Beam Energy Scaling of Ion-Induced Electron Yield From K+ lons Impact on Stainless Steel Surfaces

M. K. Covo, A. Molvik, A. Friedman, G. Westenskow, J. J. Barnard, R. Cohen, S. M. Lund, D. Grote, P. Seidl, J. W. Kwan, D. Baca, F. Bieniosek, C. M. Celata, J.-L. Vay, J. L. Vujic

May 16, 2005

Particle Accelerator Conference 2005 Knoxville, TN, United States May 16, 2005 through May 20, 2005 


\section{Disclaimer}

This document was prepared as an account of work sponsored by an agency of the United States Government. Neither the United States Government nor the University of California nor any of their employees, makes any warranty, express or implied, or assumes any legal liability or responsibility for the accuracy, completeness, or usefulness of any information, apparatus, product, or process disclosed, or represents that its use would not infringe privately owned rights. Reference herein to any specific commercial product, process, or service by trade name, trademark, manufacturer, or otherwise, does not necessarily constitute or imply its endorsement, recommendation, or favoring by the United States Government or the University of California. The views and opinions of authors expressed herein do not necessarily state or reflect those of the United States Government or the University of California, and shall not be used for advertising or product endorsement purposes. 


\title{
BEAM ENERGY SCALING OF ION-INDUCED ELECTRON YIELD FROM K+ IONS IMPACT ON STAINLESS STEEL SURFACES*
}

\author{
M. Kireeff Covo**, A. Molvik, A. Friedman, G. Westenskow, J.J. Barnard, R. Cohen, S.M. Lund, \\ and D. Grote, LLNL, Livermore, California 94550, U.S.A.
}

\author{
P. Seidl, J.W. Kwan, D. Baca, F. Bieniosek, C.M. Celata, J.-L. Vay, LBNL, 1 Cycloton Road, \\ Berkeley, California 94720 , U.S.A.
}

\author{
J.L. Vujic, UCB, 4155 Etcheverry Hall, MC 1730, Berkeley, CA 94720, U.S.A.
}

\begin{abstract}
The cost of accelerators for heavy-ion inertial fusion energy (HIF) can be reduced by using the smallest possible clearance between the beam and the wall from the beamline. This increases beam loss to the walls, generating ion-induced electrons that could be trapped by beam space charge potential into an "electron cloud", which can cause degradation or loss of the ion beam. In order to test the physical mechanism model of ioninduced electrons production we have measured the impact of $\mathrm{K}+$ ions with energies up to $400 \mathrm{KeV}$ on stainless steel surfaces near grazing incidence, using the ion source test stand (STS-500) at LLNL. The electron yield will be discussed and compared with experimental measurements from $1 \mathrm{MeV} \mathrm{K}+$ ions in the High-Current Experiment at LBNL.
\end{abstract}

\section{INTRODUCTION}

The cost of accelerators for High Energy Density Physics and Heavy Ion Fusion can be drastically reduced by increasing the fill factor. At the range of interest (beyond $60 \%$ ), the beam halo is expected to produce electrons and desorbed gas, which could move to the beam path and be ionized. The electrons produced may be trapped by the space charge beam potential. If sufficient electrons are trapped, we could lose control of the beam transport, resulting in more beam hitting the wall producing more electrons and desorbed gas. This is the beginning of the "electron cloud effect", a recognized problem in positive-charged-particle accelerator rings (see e.g. ECloud2004 [1] \& PAC2003 [2] conferences) and in the low energy $(1 \mathrm{MeV})$ region of linacs for heavy ion fusion.

\section{DESCRIPTION OF EXPERIMENT}

In order to test the physical mechanism model of ioninduced electrons production we have measured the electron yield by placing the Gas-Electron Source Diagnostics experiment (GESD) [3] at the end of a 500 kilovolt ion source test stand (STS-500) [4], which can generate a 17 microsecond duration, up to $500 \mathrm{KeV}$ and 1A pulse every few seconds.

\footnotetext{
* This work was performed under the auspices of the U.S. Department of Energy by University of California, LLNL and LBNL under contracts W-7405-Eng-48, and DE-AC03-76F00098.

** michel@nuc.berkeley.edu
}

The GESD, which is shown in Fig. 1, consists of several electrodes that are independently biased. It is designed to measure ion-induced electron emission and gas desorption from heavy-ion beams impacting a surface. The beam current passing through a small aperture hits the stainless steel target. The target is treated using LBNL ultrahigh vacuum cleaning procedures [5] and is adjustable between angles of incidence from 82 to 89.5 degrees from normal to the surface, which corresponds to $\theta$ in the Fig. 1. Between the aperture and the target a suppressor electrode prevents electrons from entering or leaving the GESD. At the end of the target a catcher is placed to capture the reflected ions. Around the target we have a grid and under the target we have a Faraday Cup (FC). If we apply correct bias to each electrode, we can measure the electron yield. The procedure consists of first placing the $\mathrm{FC}$ in the beam to measure the ion current entering the GESD, after that we move the target to a desired angle and measure the electron current leaving. The electron yield coefficient is the number of electrons produced by each ion.

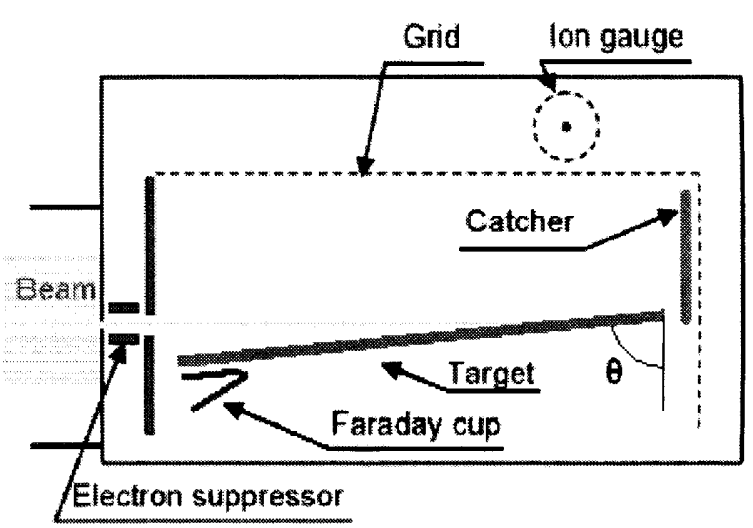

Figure 1: Gas-electron source diagnostic (GESD).

\section{RESULTS}

The energy range covered in this work is represented in Fig. 2 with gray color. At low energies (below $250 \mathrm{KeV}$ for $\mathrm{K}+$ ions) the nuclear stopping power is larger than the electronic, at higher energies the electronic component dominates. Our goal here is to check our understanding of the mechanism of ion-stimulated electron production. For that we acquire data with the GESD device using the STS 
500 facility that can operate from $\sim 50 \mathrm{KeV}$ to $500 \mathrm{KeV}$, where the nuclear stopping power begins dominating and we transition to the electronic stopping power dominance. Those data will be compared with data collected previously with the $\mathrm{HCX}$ at $1 \mathrm{MeV}$, where the electronic stopping power dominates.

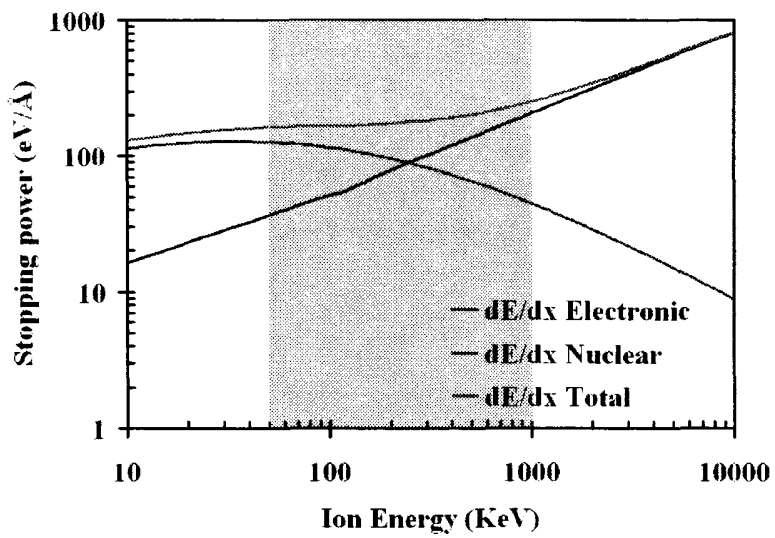

Figure 2: Stopping power for $\mathrm{K}+$ ions hitting Stainless Steel target.

Sternglass [6] developed an ion-induced electron model and derived a simple expression in which the electron yield is proportional to the electronic stopping power, as represented in Eq. 1.

$$
\gamma=K\left(\frac{d E}{d x}\right)_{e}
$$

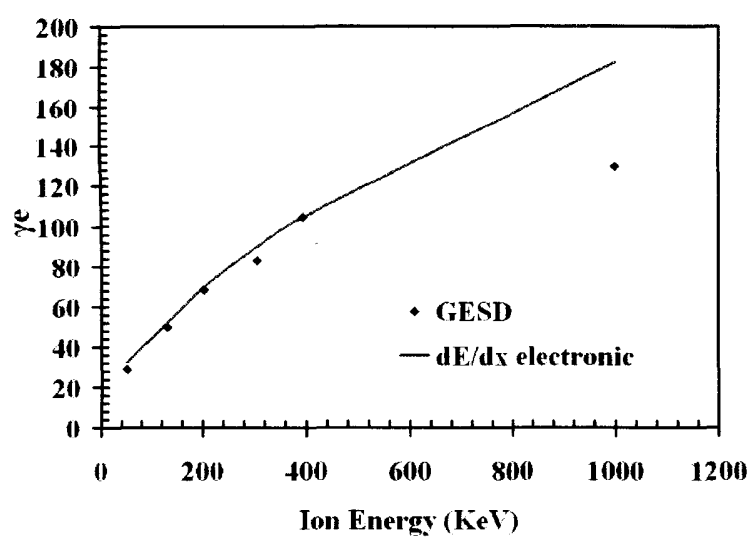

Figure 3: Electron Yield obtained with Gas-Electron Source Diagnostic at 88 degrees compared with the electronic stopping power normalized to $392.8 \mathrm{KeV}$.
Fig. 3 compares electron yield (ye) acquired with GESD at 88 degrees with the electronic stopping power from SRIM 2003 software [7] normalized to $392.8 \mathrm{KeV}$. Our result is in good agreement with the theory at lower energies, but there is a difference of $40 \%$ at higher energies, where the electronic stopping power prevails.

\section{CONCLUSIONS}

The literature has a variety of studies of ion-induced electron emission $[8,9,10]$, but they are mainly done at low or high energies, where either electronic or nuclear stopping power dominate.

Data for heavy ions over the range of medium energies where there is a transition from the predominance of nuclear to electronic stopping power is scarce. The general tendency is to extrapolate the data from light ions, applying a different correction factor $\mathrm{K}$ given in Eq. 1 .

The electron yield proportionality with ion energy is confirmed for low energies, but a difference of $40 \%$ is observed at $1 \mathrm{MeV}$. We plan to extend the analysis of the data in a further article to better explain the difference obtained. The model being developed will consider more parameters, such as the fact that almost $70 \%$ of the incident ions are backscattered and therefore do not excite the same amount of electrons.

\section{ACKNOWLEDGEMENTS}

We want to thanks Ron Beggs, Tak Katayanagi, Craig Rogers and Gary Ritchie who constructed and maintain GESD and their supervisor Ralph Ripple and William Strelo. We also want to express our gratitude to Robert Hall for his help with the STS-500 operation at LLNL. We appreciate all the support and encouragement of Grant Logan.

\section{REFERENCES}

[1] http://icfa-ecloud04.web.cern.ch/icfa-ecloud04/

[2] http://www-conf.slac.stanford.edu/pac03/

[3] A.W. Molvik et al, Phys. Rev. ST AB 7 (2004) 093202.

[4] L. Ahle et al. Laser and Particle Beams 2004 (2002) 603.

[5] K. Kennedy, LBNL Report No. LSME-323,1990.

[6] E.J Sternglass, Phys. Rev. 108 (1957) 1.

[7] J. F. Ziegler, http://www.srim.org/.

[8] E.V. Alonso et al, Phys. Rev. B22 (1980) 80.

[9] P. Thieberger et al, Phys. Rev. A 61 (2000) 042901

[10] A. Dubus et al, Nuc. Instr. and Meth. B 193 (2002) 621. 